\title{
Carcinoma de origen desconocido: diagnóstico y manejo terapéutico
}

\author{
B. Cantos Sánchez de Ibargüen ${ }^{1}$, A. Sánchez Ruiz ${ }^{1}$, C. Maximiano Alonso ${ }^{1}$, \\ A. Hurtado Nuño ${ }^{1}$, M. R. Sánchez Yuste ${ }^{2}$
}

\section{Resumen}

El carcinoma de origen desconocido se define como la existencia de enfermedad metastásica sin primario detectable al diagnóstico. Supone hasta el 3\% de las neoplasias malignas y es uno de los 10 diagnósticos de cáncer más frecuente. La definición requiere la realización de una serie de exploraciones básicas para determinar que no existe tumor primario conocido. Dentro de este heterogéneo grupo existen una serie de entidades clinicopatológicas que presentan un mejor pronóstico, ya que tienen un tratamiento específico. El esfuerzo en la búsqueda del primario irá encaminado a definir estas entidades y a saber reconocerlas. Actualmente existen diversas técnicas de inmunohistoquímica, biología molecular y radiodiagnóstico que parecen facilitar la labor al clínico a la hora de detectar el origen de los tumores, aunque ninguna de ellas es concluyente. Intentaremos analizar las distintos métodos diagnósticos, así como definir aquellas entidades que se beneficiarán de un tratamiento concreto

\section{Palabras clave:}

Cáncer. Carcinoma de origen desconocido. Inmunohistoquímica. PET. Metástasis. Diagnóstico. Tratamiento.

Oncología, 2006; 29 (3):95-106

\footnotetext{
${ }^{1}$ Servicio de Oncología Médica

${ }^{2}$ Servicio de Anatomía Patológica

Hospital Universitario Puerta de Hierro

Madrid
} 


\section{Summary}

Carcinoma of unknown primary origin is defined as the appearance of a metastatic disease where no primary tumor is detectable. It represents up to $3 \%$ of the malignant neoplasms and is among the ten most frequent cancer diagnosis. The consideration of cancer of unknown primary localization requires the performance of several basic explorations resulting insufficient to diagnose a primary tumor. Within the heterogeneous group of cancer of unknown origin, there are some clinicopathologic entities having specific treatment whose diagnosis would represent a better prognosis. It is then necessary to strive for making the diagnosis of the primary tumor, having in mind such entities. There are nowadays several techniques that may help this purpose, related with immunochemistry, molecular biology and radiodiagnosis, although even with them the results may be inconclusive. We attempt in this work to analyze the different diagnostic methods, and to define those entities which may benefit with a particular treatment.

Key words: Cancer. Unknown primary cancer. Immunohistochemistry. PET. Metastasis. Diagnosis. Treatment.

\section{Introducción}

Carcinoma de origen desconocido (COD), es el término más empleado para definir a un grupo heterogéneo de pacientes en los que no se detecta un tumor primario. En un 3-5\% de los carcinomas confirmados histológicamente no se detecta un tumor primario.

Históricamente la definición ha cambiado, según han ido variando los criterios de inclusión y las técnicas diagnósticas. En los años 70, algunos investigadores argumentaron que sólo se podía definir un carcinoma como de origen desconocido si no se encontraba el origen tras la realización de una autopsia ${ }^{1}$.

Esta definición varía de un estudio a otro, pero actualmente para llegar a definir un carcinoma de origen desconocido se requiere que el paciente presente un cáncer metastásico histológicamente documentado, en los cuales exista una detallada historia clínica, una minuciosa exploración física que incluya examen pélvico y rectal, la realización de una analítica de sangre y una bioquímica, análisis de orina y test de sangre oculta en heces, la revisión de la biopsia utilizando técnicas de inmunohistoquímica, la realización de una radiografía de tórax y un TAC abdominal y una mamografía según los casos².

Existen otras pruebas que añadirán valor al diagnóstico de estas entidades pero que varían en fun- ción del cuadro clínico de presentación. En cada caso existen detalles clínicos y patológicos únicos que requieren consideración para lograr un manejo adecuado.

El pronóstico de los pacientes con COD es muy precario, con una supervivencia media de 3-4 meses, sólo un $25 \%$ de los pacientes están vivos al año y el $10 \%$ a los 5 años. Aunque la mayoría de las enfermedades son refractarias a los tratamientos sistémicos, existen una serie de presentaciones clínicas con un pronóstico más favorable.

Con el aumento en el número de modalidades diagnósticas, los clínicos deben determinar cuanto esfuerzo diagnóstico es necesario antes de aceptar un diagnóstico de COD y se debe considerar también si un diagnóstico más preciso tendrá repercusión sobre el tipo de tratamiento a aplicar y sobre la supervivencia global del enfermo.

\section{Epidemiología}

Según datos epidemiológicos, el carcinoma de origen desconocido supone el 2,3-4,2\% de todos los cánceres humanos ${ }^{3}$. En un $15-25 \%$ de los casos, no se detecta el tumor primario ni en las autopsias. La incidencia anual ajustada por edad es de 7-12 casos por 100000 habitantes por año en USA y de 18-19 
casos por 100000 habitantes por año en Australia. Representa la cuarta causa de muerte por cáncer en hombres y mujeres (en conjunto), lo que supone que es causa más frecuente de muerte que el Linfoma de Hodgkin.

La edad media de presentación son los 60 años y es ligeramente superior la incidencia en varones.

\section{Diagnóstico}

El diagnóstico irá enfocado a reconocer el subgrupo de pacientes dentro del heterogéneo grupo de carcinoma de origen desconocido que se beneficiará de recibir un tratamiento específico, que les podría mejorar la supervivencia.

\section{Historia clínica}

Es fundamental realizar una historia clínica completa haciendo hincapié en el resultado de biopsias previas, cirugías y lesiones resecadas, así como en la historia familiar oncológica que puede determinar una predisposición familiar al carcinoma de colon hereditario no polipósico o al carcinoma de mama².

\section{Exploración física}

El examen físico debe incluir una palpación tiroidea, de las mamas, de los ganglios linfáticos, de la próstata y un tacto rectal. En las mujeres está recomendada la exploración ginecológica. La presencia de una adenopatía supraclavicular izquierda (nódulo de Virchow) o una adenopatía periumbilical (nódulo de la hermana Maria José) podrían orientar a un origen gastrointestinal.

\section{Evaluación anatomopatológica}

Es esencial la comunicación entre clínico y patólogo para poder asegurar un beneficio en el trabajo. Otro punto fundamental es que el patólogo disponga de material suficiente para poder realizar los distintos estudios. Los diagnósticos que se llevan a cabo basados en una citología (PAAF) normalmente no dispondrán de material para realizar las distintas técnicas de inmunohistoquímica.

El patólogo podrá realizar el diagnóstico hasta en un $20 \%$ de los casos.
TABLA I

\section{Clasificación histopatológica}

Adenocarcinoma

Bien/moderadamente diferenciado

Pobre/indiferenciado

Escamoso

Neoplasias indiferenciadas

(tumores neuroendocrinos, linfomas, tumor germinal

melanoma, sarcoma, tumores embrionarios...)

\section{A. Hematoxilina-Eosina}

El examen al microscopio puede básicamente describir la morfología celular y la diferenciación celular. Con esta técnica los tumores serán clasificados tal y como se detallan en la Tabla I.

\section{B. Inmunohistoquímica}

Los marcadores inmunohistoquímicos (IHQ) ayudan a definir una línea tumoral cuando se utilizan anticuerpos mediados por la peroxidasa contra antígenos específicos del tumor. No existe ningún test de inmunohistoquímica que sea $100 \%$ específico, por lo que los marcadores de IHQ sólo serán un dato más que junto con el cuadro de presentación y los estudios radiológicos orientarán sobre un posible origen. La Tabla II muestra un panel de screening de las tinciones de IHQ más utilizadas.

Las citoqueratinas (CK) más empleadas en el carcinoma de origen desconocido son la CK 7 y CK 20. La citoqueratina 20 es una CK de bajo peso molecular que generalmente se expresa en el epitelio gastrointestinal, urotelio y células de Merckel. La citoqueratina 7 se encuentra en el cáncer de pulmón, ovario, endometrio y mama y no en tumores del tracto gastrointestinal bajo ${ }^{4}$. La combinación de estas 2 citoqueratinas orientan hacia distintos tumores, como se pone de manifiesto en la Tabla III.

TTF-1 (factor de trascripción tiroidea) es una proteína nuclear que interviene en la activación de la trascripción durante la embriogénesis en el tiroides, diencéfalo y epitelio respiratorio. Es típicamente positivo en los carcinomas de pulmón y tiroides. Aproximadamente el 68\% de los adenocarcinomas de pulmón serán positivos y el $25 \%$ de los carcinomas escamosos ${ }^{2}$.

Los anticuerpos contra proteínas específicas de las células del epitelio respiratorio A y B, en ocasio- 
TABLA II

Diagnóstico diferencial de carcinoma pobremente diferenciado con técnicas de inmunoperoxidasas

\begin{tabular}{|c|c|c|c|c|c|c|}
\hline Tipo tumor & Citoqueratinas & $\begin{array}{c}\text { Antígeno } \\
\text { membrana epitelial }\end{array}$ & $\begin{array}{c}\text { Antígeno } \\
\text { leucocitario común }\end{array}$ & S 100 proteína & Vimentina & $N S E^{l}$ \\
\hline Carcinoma & + & + & - & - & - & $+/-$ \\
\hline Linfoma & - & $+/-$ & + & - & - & - \\
\hline Melanoma & - & - & - & + & + & + \\
\hline Sarcoma & - & $+/-$ & - & - & + & - \\
\hline Neuroendocrino & + & + & - & - & - & + \\
\hline Germinal & + & + & - & - & - & - \\
\hline Próstata & + & + & - & - & - & - \\
\hline Mama & + & + & - & - & - & - \\
\hline Tipo tumor & $\mathrm{HCG}^{2}$ & $\mathrm{AFP}^{3}$ & $\mathrm{PSA}^{4}$ & $\mathrm{RE}^{5}$ & Desmina & $\begin{array}{c}\text { Cromogranina } \\
\text { Sinaptofisina }\end{array}$ \\
\hline Carcinoma & $+/-$ & $+/-$ & $+/-$ & $+/-$ & - & $+/-$ \\
\hline Linfoma & - & - & - & - & - & - \\
\hline Melanoma & - & - & - & - & - & - \\
\hline Sarcoma & - & - & - & - & $t^{\mathrm{a}}$ & - \\
\hline Neuroendocrino & - & - & - & - & - & + \\
\hline Germinal & + & $+/-$ & - & - & - & - \\
\hline Próstata & - & - & + & - & - & - \\
\hline Mama & - & - & - & + & - & - \\
\hline $\begin{array}{l}\text { Enolasa neuroespecí } \\
\text { Gonadotropina coric } \\
\text { Alfafetorpteina }\end{array}$ & $\begin{array}{l}\text { f́ica } \\
\text { Snica humana }\end{array}$ & & $\begin{array}{l}{ }^{4} \text { Antígeno es } \\
{ }^{5} \text { Receptor es } \\
{ }^{\text {a }} \text { Rabdomios }\end{array}$ & $\begin{array}{l}\text { ecífico de próstata } \\
\text { ogénico } \\
\text { coma }\end{array}$ & & \\
\hline
\end{tabular}

TABLA III

Orientación diagnóstica según la expresión de citoqueratinas

\begin{tabular}{|lll|}
\hline & Citoqueratina 20 positiva & Citoqueratina 20 negativa \\
\hline Citoqueratina 7 positiva & Urotelial-transicional & Tiroides \\
& Biliar & Pulmón \\
& Páncreas & Ovario no mucinoso \\
& Ovario mucinoso & Mama \\
& & Biliar \\
& & Páncreas \\
& & Endometrio \\
& & Cervix \\
& & Glándula salival \\
& & Próstata \\
& Colorectal & Riñón \\
& Gástrico & Adeno hepático \\
& Ovario mucinoso & Escamoso pulmón \\
& Cel Merckel & Cabeza y cuello \\
\hline
\end{tabular}

nes son útiles para el diagnóstico de carcinoma de pulmón de célula no pequeña. Existen estudios (Bejarano y colaboradores $)^{5}$ en los que se detectan hasta en el $54 \%$ y $63 \%$ de los cánceres de pulmón.
El antígeno Hep par 1 está expresado en los hepatocitos tanto benignos como malignos, por lo que el anticuerpo monoclonal contra este antígeno orientará hacia un hepatocarcinoma. No existe ningún mar- 
cador IHQ que diferencie entre el colangiocarcinoma y las metástasis de adenocarcinoma ${ }^{6}$.

GCDFP-15 (también conocida como proteína inducida por la prolactina) es un marcador de diferenciación apocrina y es expresado específicamente en pacientes con cáncer de mama. También se expresa en glándulas salivales, piel, glándulas bronquiales, próstata y vesículas seminales.

URO III, citoqueratinas de alto peso molecular, trombomodulina y CK 20 son marcadores del epitelio urotelial.

\section{Microscopía electrónica}

Es una técnica cara y que no está disponible en todos los centros. Sólo es recomendable en el caso de tumores poco diferenciados. En ciertas ocasiones puede ser útil para diferenciar linfomas de carcinoma, adenocarcinoma de carcinoma escamoso y para diferenciar tumores neuroendocrinos o melanomas ${ }^{3}$.

\section{Diagnóstico molecular}

Es de uso muy limitado, ya que sólo se han identificado algunas alteraciones cromosómicas específicas para tumores.

- $12 \mathrm{i}(12 \mathrm{p})$ o delección $12 \mathrm{p}$ : se asocia a carcinomas testiculares o germinales.

- $\mathrm{t}(11,22)$ (q24;q12): se observa en todos los neuroepiteliomas periféricos y sarcoma de Ewing.

- $\mathrm{t}(8,14)$ (q24;q32): se relaciona con linfomas no hodgkin.

- t (3;13): se observa en el rabdomiosarcoma alveolar.

- Delección 3p: se encuentra en los carcinomas de pulmón de célula pequeña.

- t (15:19): es una traslocación que puede encontrarse en los carcinomas pobremente diferenciados de la línea media (nasofaringe, mediastino, vejiga) en pacientes jóvenes. Este tipo de alteración cromosómica resulta en una fusión de oncogenes BRD4-NUT que confiere un peor pronóstico a pesar de emplear quimioterapias agresivas ${ }^{7,8}$.

\section{Marcadores tumorales serologicos}

Únicamente es aceptado con valor diagnóstico el PSA en varones con adenocarcinoma y metástasis óseas. La $\beta$-HCG (gonadotropina coriónica humana $\beta)$ y AFP (alfafetoproteína) pueden indicar la presencia de un tumor germinal sobre todo en varones jóvenes con carcinoma indiferenciado que se manifiesten en la línea media.

La AFP también es útil en el diagnóstico del hepatocarcinoma.

Otros marcadores tales como el CEA, Ca 125, Ca 19.9, Ca 15.3 no son útiles para establecer la localización del primario tumoral. La mayoría de estos marcadores serológicos no son específicos y suelen tener algún valor en el pronóstico de la enfermedad pero no en le diagnóstico de la misma ${ }^{2}$. Existe una publicación de Koch y McPherson en la que revisaron 32 pacientes con COD, en el análisis que realizan concluyen que pacientes con CEA > $10 \mathrm{ng} / \mathrm{ml}$ sugiere que el origen esté en mama u ovario, aunque si existen metástasis hepáticas este mismo valor orienta hacia un origen pancreático o de intestino 9 . Estos datos no han sido comprobados por otros estudios publicados.

\section{Estudios radiológicos}

\section{A. Rx tórax y TAC abdominal}

Están incluidas dentro de los test de rutina a realizar en todo paciente con un carcinoma de origen desconocido. Basado en una serie de autopsias, la radiografía de tórax es capaz de diferenciar entre un primario de pulmón y metástasis solamente en un tercio de $\operatorname{los} \operatorname{casos}^{10}$. Un pequeño estudio retrospectivo ${ }^{11}$ demostró que en un $38 \%$ de los pacientes que presentaban radiografías de tórax inespecíficas o negativas, el TAC de tórax sí detectaba el tumor primario. Es recomendable la realización de un TAC torácico cuando la histología oriente a un posible origen pulmonar. La tendencia habitual en la clínica es a la realización de un TAC toracoabdominal, que además de detectar el primario en alguna ocasión servirá para discernir el sitio más apropiado para realizar la biopsia.

\section{B. Mamografía y otros estudios mamarios}

Sólo se indicará de rutina en mujeres con adenocarcinoma y afectación exclusivamente axilar (sensibilidad del $22 \%)^{12}$ o metástasis a distancia. Si la sospecha de primario de mama es muy elevada, bien sea por la presentación clínica o por la presencia de 
TABLA IV

Estudios realizados en pacientes diagnosticados de carcinoma de origen desconocido para valorar la rentabilidad del PET como prueba diagnóstica en esta entidad

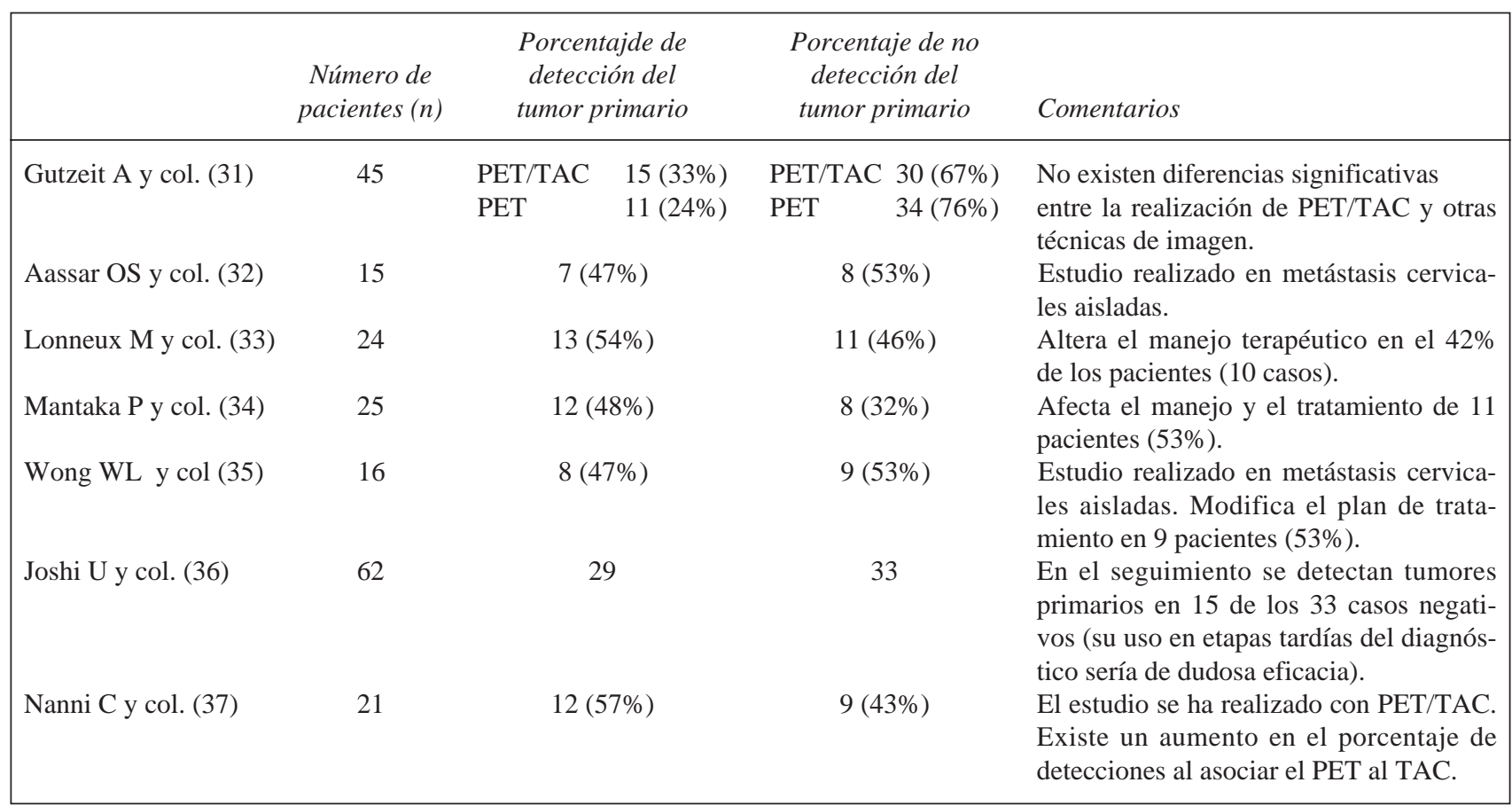

una historia familiar concordante y los estudios radiológicos y ecográficos no son concluyentes podría estar indicada la realización de una RMN (resonancia magnética nuclear) de la mama, aunque esta establecerá el diagnóstico en muy contadas ocasiones.

\section{Resonancia magnética nuclear}

El papel de la RMN excepto el descrito con anterioridad para la mama, es escaso. Generalmente su uso queda relegado a pacientes en los que exista una contraindicación para la realización de un TAC.

\section{FDG-PET}

El PET (tomografía por emisión de positrones) utiliza un marcador radiológico para revelar información tanto anatómica como metabólica en varios tejidos y órganos. El marcador utilizado es un análogo de la glucosa, 2-(fluorina-18)-fluoro-2-deoxiD-glucosa (FDG), las células tumorales utilizan la glucosa más rápidamente que las células normales, este análogo al ser no degradable se acumula más y será localizado por la emisión de positrones.
La identificación del tumor primario tras una exhaustiva búsqueda aún continua siendo un problema a pesar de los recientes avances en las técnicas de imagen. El PET ha ganado una importante posición en la evaluación de los tumores de origen desconocido, sobre todo cuando no se ha detectado el primario tras la realización de las exploraciones rutinarias.

En 1994 comienzan a publicarse estudios que evalúan la utilidad del PET-FDG en los carcinomas de origen desconocido para detectar el tumor primario. La mayoría de estos estudios aseguran que el PET-FDG detecta el tumor primario en alrededor de un $40 \%$ de los pacientes con COD en los que los procedimientos diagnósticos convencionales resultaron negativos (Tabla IV).

La utilidad del FDG-PET en las imágenes del carcinoma de cabeza y cuello ha sido ampliamente demostrada $^{2}$. Numerosos estudios avalan el valor del FDG-PET en detectar, estadiar y evidenciar recurrencias de la enfermedad trás la radioterapia ${ }^{13}$. Rusthoven y col. ${ }^{14}$ realizan una revisión de 16 estudios que evalúan el papel del FDG-PET en la detección de tumores de origen desconocido, recogen 
datos de 302 pacientes que presentan metástasis cervicales de primario desconocido y concluyen que el FDG-PET tiene una sensibilidad de hasta el $88 \%$ en detectar tumores primarios, en aproximadamente el $25 \%$ de los casos detectará el tumor primario en pacientes con metástasis cervicales en los que no se había detectado el tumor primario con las técnicas habituales y hasta en un $27 \%$ puede detectar metástasis a distancia o regionales desconocidas. Esto implica cambios en los tratamientos, ya que se reduce el campo de radioterapia e incluso se evitará el tratamiento local agresivo en determinados pacientes.

Se han realizado múltiples estudios para determinar la utilidad el FDG-PET en las otras formas de presentación del carcinoma de origen desconocido. Existe un meta-análisis de Delgado -Bolton ${ }^{15}$ que concluye que tiene una especificidad intermedia (0.71) y una elevada sensibilidad (0.87) con pocos resultados falsos negativos. Existe suficiente evidencia para recomendar su uso en pacientes candidatos a un tratamiento local o regional, ya que la detección de otras localizaciones modificará el tipo de tratamiento a recibir, sin embargo no existe evidencia científica para determinar la utilidad en pacientes que presenten múltiples metástasis, ya que no se ha comparado si el cambio en el tipo de tratamiento según el tumor sospechado influirá en la supervivencia del enfermo. En casi todos los estudios publicados, el FDG-PET se ha realizado tras las técnicas diagnósticas habituales, por lo que no se puede concluir que el FDG-PET pueda sustituir a las técnicas utilizadas hasta el momento y únicamente se recomienda su uso como parte del algoritmo diagnóstico del carcinoma de origen desconocido ${ }^{17}$.

El punto débil de esta técnica continua siendo su baja especificidad, ya que cualquier enfermedad inflamatoria, la actividad muscular, los ganglios linfáticos reactivos... pueden dar un falso positivo. Probablemente la combinación del PET-TAC disminuya de forma considerable el número de falsos positivos, aunque esto está pendiente de confirmación con los distintos ensayos que se encuentran en marcha.

Ya en el año 2000, en la $3^{a}$ Conferencia Interdisciplinar de Consenso Alemana, "Onko-PET III", que actualiza la evaluación de la eficacia clínica de la PET-FDG en cada una de las indicaciones oncológicas, sitúa en el grado 1a la indicación de la PETFDG en el COD, que se considera como uso clínico establecido $^{17}$. En España se acepta dentro del procedimiento de "Uso Tutelado" cuando se espere que la PET-FDG pueda cambiar el manejo del paciente.

\section{E. Técnicas invasivas}

Broncoscopia, endoscopia y colonoscopia no se solicitarán de forma rutinaria en pacientes asintomáticos. Su uso debe ser valorado individualmente según la forma de presentación de la enfermedad en cada paciente ${ }^{8}$.

Una triple endoscopia que incluya laringoscopia, broncoscopia y esofagoscopia sí se debe realizar de rutina en aquellos pacientes que se presenten con adenopatías cervicales. Recomendaremos la realización de una colonoscopia en pacientes que presenten metástasis hepáticas resecables con histología de adenocarcinoma.

\section{Técnicas en desarrollo}

El diagnóstico molecular en el cáncer es una técnica prometedora. La existencia de una base de datos de expresión génica para diversas enfermedades malignas puede ser de gran ayuda. Se han comenzado ha realizar estudios basados en identificar un subgrupo de genes cuya expresión sería específica del tejido de origen así como genes cuya expresión estuviera elevada en los pacientes con cáncer. No existen todavía datos suficientes para avalar esta técnica. Necesitaríamos crear un perfil de genes de los tumores primarios conocidos que representaran al tipo de tumor que está presente en la población a estudio.

\section{Tratamiento}

En general los pacientes con carcinoma de origen desconocido tienen una supervivencia media de 6-9 meses, la gran mayoría presentan una enfermedad diseminada que en muchas ocasiones es quimiorresistente, sin embargo existen unos subtipos clínicos que tiene un mejor pronóstico en los que podría aplicarse un tratamiento potencialmente curativo.

El exhaustivo trabajo realizado en el diagnóstico de estos enfermos únicamente tiene como meta el ser capaz de discernir aquellos casos que pueden tener un mejor pronóstico. 


\section{Subgrupos de buen pronóstico}

\section{A. Metástasis cervicales de carcinoma escamoso}

En algunas guías se recomienda la tonsilectomía bilateral si las amígdalas no se han extraído previamente. El manejo terapéutico será similar al de un carcinoma de cabeza y cuello localmente avanza$\mathrm{do}^{18}$.

Es importante discernir qué pacientes con afectación nodal supraclavicular tendrán con más posibilidad un tumor pulmonar o de tracto gastrointestinal que un carcinoma de cabeza y cuello.

Entre las distintas opciones de tratamiento se encuentran la disección radical cervical, la irradiación y la combinación de ambas técnicas. Existen algunos estudios que apoyan un tratamiento agresivo bilateral cervical con irradiación total de la mucosa ${ }^{19}$ lo que se asocia con una elevada toxicidad y otros argumentan la limitación de la radioterapia a la zona cervical ipsilateral ${ }^{20}$. Se han descrito controles locales prolongados hasta en el 50-75\% de los casos según las series. Las tasas de supervivencia a 5 años van desde el $30 \%$ al $50 \%$. Como era de esperar el volumen tumoral es un importante factor pronóstico, así como la afectación nodal a múltiples niveles o la extensión extracapsular, estas situaciones requerirán tratamientos combinados de elevada agresividad.

El impacto de la quimioterapia en este tipo de pacientes permanece indefinido. El hecho de que el tratamiento con quimioradioterapia concomitante esté cogiendo fuerza en los tratamientos de los carcinomas avanzados de cabeza y cuello sugiere que pacientes con afectación ganglionar $\mathrm{N}_{2} \mathrm{O} \mathrm{N}_{3}$ por un carcinoma de origen desconocido podrían beneficiarse de este tipo de tratamiento ${ }^{3}$.

\section{B. Síndrome de célula germinal extragonadal}

Este síndrome fue descrito por primera vez por Richardson en 1981. Las características clínicas incluyen; varón joven (menos de 50 años de edad), tumor predominantemente localizado en la línea media, múltiples nódulos pulmonares o afectación ganglionar, corto periodo de latencia desde el inicio de los síntomas hasta acudir al médico para valoración, historia de crecimiento tumoral rápido, niveles elevados de AFP o $\beta$-HCG o células positivas para dichas tinciones por inmunohistoquímica, presencia de gránulos neuroendocrinos y tumores que fueron muy sensibles a quimioterapia o radioterapia. La mayoría de los pacientes no muestran todas las alteraciones.

Los pacientes que expresen alguna de estas características deben tratarse con un régimen de quimioterapia basado en platino. El tratamiento es similar a los carcinomas germinales con mal pronóstico.

Se han descrito supervivencias libres de enfermedad prolongadas. Con los esquemas de platino se consigue una tasa de respuesta que oscila entre el $50-70 \%$ con más de un $25 \%$ de respuestas completas y supervivencias sin enfermedad a largo plazo de un $16 \% \%^{3,18,21}$.

\section{Carcinomatosis peritoneal en mujer}

Las mujeres que debutan con una carcinomatosis peritoneal diseminada conllevan un diagnóstico de presunción de tumor ovárico. Ocasionalmente podría tratarse de un tumor mamario o gastrointestinal aunque esta forma de presentación es mucho menos frecuente.

La carcinomatosis peritoneal primaria, también conocida como carcinoma seroso multifocal extraovárico o carcinoma serosopapilar peritoneal tiene una presentación clínica muy similar al ovario. La única diferencia es que en la carcinomatosis peritoneal no se objetivan lesiones visibles en los ova$\operatorname{rios}^{18}$. Puede presentarse tras ofoorectomía y en la anatomía patológica pueden observarse rasgos papilares y cuerpos de psamoma. Las metástasis suelen estar limitadas a la cavidad peritoneal y el $\mathrm{Ca} 125$ suele elevarse.

Si la histología confirma la existencia de un adenocarcinoma seroso papilar el manejo debe ser similar al del carcinoma de ovario en estadio III de la FIGO con esquema de quimioterapia basadas en taxanos/platino. La tasa de respuestas completas en los distintos estudios varía entre el 10-39\% con una supervivencia media de 16 meses y una supervivencia a largo plazo (5 años) del 16\%.

El manejo óptimo de estas pacientes debería incluir una agresiva citorreducción con resección de toda la enfermedad visible (> de 1 centímetro) y quimioterapia adyuvante con un esquema de platino asociado o no a un taxano ${ }^{18,22,23}$.

La quimioterapia intraperitoneal o la radioterapia no han demostrado beneficios clínicos. 


\section{Mujer con metástasis axilar aislada (adenocarcinoma)}

Generalmente el manejo de estas pacientes va a ser similar al del estadio II o III de mama.

La evaluación patológica inicial de un ganglio axilar afectado por adenocarcinoma debe incluir la realización de la determinación del nivel de receptores estrogénicos y de progesterona. Si el ganglio linfático presenta una positividad marcada para estos receptores orientarán al origen mamario del mismo.

Si la axila es la única localización afectada, estas pacientes deben tratarse con quimioterapia adyuvante, como si se tratase de un estadio II de cáncer de mama, incluyendo el Tamoxifeno para aquellas pacientes que tengan receptores hormonales positivos. Enfermas con enfermedad $\mathrm{N}_{2}$ (ganglios axilares fijos) deben recibir tratamiento como si se tratase de un estadio III de cáncer de mama, incluyendo quimioterapia neoadyuvante y el Tamoxifeno en caso de que fuese necesario ${ }^{3,18}$.

Aquellas pacientes que no respondan o que sean muy mayores pueden tratarse con irradiación radical y continuar con Tamoxifeno en el caso de que los receptores sean positivos

Un punto de mayor controversia en el tratamiento es el manejo quirúrgico de estas pacientes. El tratamiento habitual en estos casos era la mastectomía radical modificada con una meticulosa evaluación patológica de la pieza resecada. Otra de las opciones quirúrgicas sería la realización de una disección axilar y observación. Esta estrategia se basaba en que en determinadas ocasiones el tumor primario no daba la cara y que el pronóstico de la enferma estaba marcado por la afectación ganglionar ${ }^{18}$.

En la actualidad existe mayor tendencia a la conservación de la mama. La terapia locorregional se basaría en una irradiación externa de toda la mama y una disección axilar. En diversos estudios no se han encontrado diferencias en la supervivencia entre aquellas que se someten a mastectomía y aquellas a las que se les realiza disección axilar y radioterapia ${ }^{24,25}$.

Las tasas de supervivencia a 5 y 10 años son del 75 y $60 \%$, respectivamente ${ }^{3}$.

\section{E. Metástasis inguinal aislada de carcinoma escamoso}

$\mathrm{Su}$ origen suele ser el área genital o anal/rectal.
En mujeres, se recomienda un examen cuidadoso de la vulva, vagina y cuello uterino. El tratamiento de elección es la disección ganglionar con o sin radioterapia asociada. En algunos pacientes se han descrito supervivencias prolongadas con tratamiento locorregional ${ }^{26}$.

\section{F. Carcinoma neuroendocrino de origen desconocido}

Esta entidad podría dividirse en tres subgrupos ${ }^{3}$, el primer subgrupo tiene una histología similar al carcinoide típico o a los tumores de islotes pancreáticos. Son indolentes y generalmente el crecimiento es muy lento. Suelen metastatizar al hígado y puede existir o no la producción de una hormona. El tratamiento es similar al del tumor carcinoide de origen conocido, quimioterapia u octreótido en pacientes con producción hormonal sintomática.

El segundo subgrupo son aquellos con carcinoma metastásico de célula pequeña, que aunque no presenten un primario pulmonar documentado se tratarán como tal, con un esquema de quimioterapia basada en platino. La supervivencia media es de 12 meses y en algún caso se han documentado supervivencias prolongadas en pacientes con enfermedad limitada y respuesta completa tras tratamiento con quimioterapia y radioterapia.

El tercer subgrupo estaría formado por pacientes con carcinoma pobremente diferenciado con rasgos neuroendocrinos que se identifican por microscopia electrónica o por inmunohistoquímica. Este subgrupo puede presentar una amplia sensibilidad a la quimioterapia. En diversos estudios las tasas de respuestas a esquemas de platino o esquemas basados en carboplatino/paclitaxel son de entre un 50 y un $70 \%$ con más del $25 \%$ de respuestas completas ${ }^{21,27}$.

\section{F. Varón con metástasis óseas blásticas y elevación del PSA}

En estos pacientes se debe considerar que existe un carcinoma de próstata metastásico e iniciar un tratamiento hormonal.

\section{G. Metástasis única de pequeño tamaño}

Existe un pequeño número de pacientes que presentan una metástasis única tras una completa evaluación clínica y radiológica. El tratamiento debería 
ser definitivo y local, es decir cirugía y/o radioterapia. El tipo de tratamiento debería estar guiado por la localización tumor. Muchos de estos pacientes se beneficien clínicamente de este tipo de procedimientos.

\section{Subgrupo de mal pronóstico}

El grupo de pacientes que presentan el subgrupo favorable es una minoría. La gran mayoría de estos pacientes no se incluyen en estos subgrupos y presentan un peor pronóstico, con supervivencias medias de entre 4 y 7 meses. La quimioterapia sistémica se emplea para controlar los síntomas y en un intento de mejorar la supervivencia aunque no se ha identificado un régimen estándar.

Para mejorar el precario pronóstico de estos enfermos, los estudios realizados en los últimos años, han insistido en la introducción de nuevos agentes citotóxicos con amplio espectro de actividad clínica, tales como la gemcitabina o los taxanos. Diferentes esquemas de quimioterapia con taxanos/platinos han resultado ser positivas, produciendo las tasas de respuesta más altas observadas, con supervivencias medias de 8-9 meses. Sacar conclusiones definitivas de estos estudios es difícil dada la heterogenicidad de este tipo de población y de la naturaleza retrospectiva de la comparación ${ }^{3}$.

Numerosos ensayos fase II han demostrado que los esquemas de quimioterapia en combinación tienen una modesta actividad en este grupo de pacien$\operatorname{tes}^{28,29}$.

Culine y col..$^{30}$ compara las respuestas a un esquema clásico de quimioterapia (cisplatino-gemcitabina) frente a esquemas con nuevos fármacos (cisplatino-irinotecan), ambos han demostrado eficacia frente al tumor, aunque la supervivencia media sigue siendo corta. No se ha demostrado que los nuevos fármacos hayan presentado un avance en el tratamiento de los COD.

Los pacientes que sean muy mayores o que presenten muy mal estado general son mucho mejor manejados con cuidados paliativos.

\section{Conclusión}

Pocas enfermedades neoplásicas crean tanta ansiedad como el diagnóstico de carcinoma de origen desconocido. En primer lugar el médico someterá al enfermo a una larga lista de exploraciones complementarias con el fin de determinar un tumor primario, sin que esto se consiga en la mayoría de los casos. Es importante determinar qué exploraciones son las que aportarán un beneficio clínico en un enfermo determinado y recordar el papel fundamental del patólogo en asociación con el clínico, en el diagnóstico. En segundo lugar ocurre una frustración y confusión a nivel del paciente al no llegar a un diagnóstico de un tumor primario.

El manejo terapeútico en la oncología moderna se basa en la identificación del tumor primario, por lo que enfrentarnos a este diagnóstico nos obligará a tomar decisiones terapeúticas sobre las que no existe un estándar establecido. La diferenciación de los subgrupos de buen pronóstico aportará un beneficio a dichos enfermos al recibir un tratamiento específico que aporta un aumento de la supervivencia. El tratamiento de los pacientes con carcinoma de origen desconocido con pronóstico desfavorable en el momento actual, aporta escasos beneficios. Los estudios deben ir enfocados a determinar que esquemas de quimioterapia pueden aportar el máximo beneficio con el mínimo de toxicidad posible.

Correspondencia:

Dra. B. Cantos Sánchez de Ibargüen

Servicio de Oncología Médica

Hospital Universitario Puerta de Hierro

San Martín Porres, 4

E-28035 Madrid 


\section{Bibliografía}

1. Holmes FF, Fouts JL. Metastasic cancer of unknown primary site. Cancer 1970, 26: 816-820.

2. Varadhachary GR, Abbruzzese JL, Lenzi R. Diagnostic strategies for unknown primary cancer. Cancer 2004, 100(9): 1776-1785.

3. Pavlidis N, Briasoulis E, Hainsworth J, Greco FA. Diagnostic and theraoeutic management of cancer of an unknown primary. Eur J Cancer 2003; 39: 1990-2005.

4. Rubin BP, Skarin AT, Pisick E, Rizk M, Salgia R. Use of cytokeratins 7 and 20 in determinig the origin of metastasic carcinoma of unknown primary with special emphasis on lung cancer. Eur J Cancer 2003; 39: 1990-2005.

5. Bejarano PA, Baughman RP, Biddinger PW et al. Surfactant proteins and thyroid transcription factor 1 in pulmonary and breast cancer. Mod Pathol 1996; 445-452.

6. Lau SK, Prakash S, Geller SA, Alsabeh R. Comparative inmunohistochemical profile of hepatocellular carcinoma, cholangiocarcinoma and metastasic adenocarcinoma. Hum Pathol 2002; 33: 1175-1181.

7. French CA, Kutok JL, Faquin WC et al. Midline carcinoma of children and young adults with NUT rearrangement. J Clin Oncol 2004; 22: 4135.

8. Dennis JL, Oien KA. Hunting the primary: novel strategies for defining the origin of tumours. J Pathol 2005; 205: 236-247.

9. Koch M, McPherson TA. Carcinoembryonic antigen levels as an indicator of the primary site in metastasic disease of unknown origin. Cancer 1981; 48(5): 1242-1244.

10. Le Chevalier T, Cvitkovik E, Coille P et al. Early metastasic cancer of unknown origin at presentation. A clinial study of 302 consecutive autopsied patients. Arch Intern Med 1998; 148: 2035-2039.

11. Latief KH, White CS, Protopapes Z, Attar S, Krasna MJ. Search for a primary lung neoplasm in patient with brain metastasis; is the chest radiograph sufficient?. AJR Am J Roenhtgenal 1997; 168: 1339-1344.

12. Baron PL, Moore MP, Kinne DW et al. Occult breast cancer presenting with axillary metastases. Updated management. Arch Surg 1990; 125: 210-214.

13. Di Martino E, Nowak B, Hassan HA et al. Diagnosis and staging of head and neck cancer: a comparision of moderm imaging modalities (positron emission tomography, computed tomography, color-coded duplex sonography) with panendoscopìc and histopathologic findings. Arch Otolaryngol Head and Neck Surg 2000; 126: 1457-1461.

14. Rusthoven KE, Koshy M, Paulino A. The role of fluorodeoxyglucose positron emission tomography in cervical lymph node metastases from unknown primary tumor. Cancer 2004; 101: 2641-2649.

15. Delgado-Bolton RC, Fernandez-Perez C, Gonzalez-Mate A et al. Meta-analysis of the performance of $18 \mathrm{~F}-\mathrm{FDG}$ PET in primary tumor detection in unknown primary tumors. J Nucl Med 2003; 44: 13C1-13C14.
16. Technology Evaluation Center. FDG PET to manage patients with an occult primary carcinoma and metastasis outside the cervial lymph node 2002: 17 (14).

17. Reske SN, Kotzerke J. FDG-PET for clinical use. Results of the 3rd German Interdisciplinary Consensous Conference, «Onko-PET III», 21 july and 19 september 2000. Eur J Nucl Med 2001; 28: 1707-1723.

18.-Chorost MI, Lee MC, Yeoh CB et al. Unknown primary. J Surg Oncol 2004; 87: 191-203.

19. Reddy SP, Marks JE. Metastatic carcinoma in the cervical lymph node from an unknown primary site. Results of bilateral neck plus mucosal irradiation vs ipsilateral neck irradiation. Int J Radiat Oncol Biol Phys 1997; 37: 797802.

20.-Nieder C, Gregoire V, Ang KK et al. Cervical lymph node metastases from occult squamous cell carcinoma: cut down a tree to get an apple. Int J Radiat Oncol Biol Phys 2001; 50: 727-733.

21. Hainsworth J, Johnson DH, Greco FA. Poorly differentiated neuroendocrine carcinoma of unknown primary: identification of a treatable subset. Ann Oncol 1990; 1: 119-122.

22. Chen KT, Flam MS. Peritoneal papillary serous carcinoma with long term survival. Cancer 1986; 58: 1371-1373.

23. Ransom DT, Patel SR, Keeney GI et al. Papillary serous carcinoma of the peritoneum. Cancer 1990; 66: 1091-1094.

24. Ellerbroek N, Holmes F, Singletary E et al. Treatment of patients with isolated axillary nodal metastasis from an occult primary carcinoma consistent with breast origin. Cancer 1990; 66: 1461-1462.

25. Fisher B, Anderson S, Redman CK et al. reanalysis and results after 12 years of follow-up in a randomized clinical trial comparing total mastectomy with lumpectomy with or without irradiation in the treatment of breast cancer. $\mathrm{N}$ Engl J Med 1995; 333: 1456-1461.

26. Guarischi A, Keane TJ, Einakim T. Metastatic inguinal nodes from an unknown primary neoplasm. A review of 56 cases. Cancer 1987; 19: 572-577.

27. Moertel CG, Kvols LK, O’Connell MJ, Rubin J. Treatment of neuroendocrine carcinoma with combined etioposide and cisplatin: evidence of major therapeutic activity in the anaplastic variant of these neoplasm. Cancer 1991; 68: 227-233.

28. Dowell JE, Garret AM, Shyr Y, Johnson DH, Hande KR. A randomized phase II trial in patients with carcinoma of an unknown primary site. Cancer 2001: 91(3): 592-597.

29. Assersohn L, Norman AR, Cunningham D et al. A randomized study of protrated venous infusion of 5-fluorouracil (5-FU) with or without bolus mitomycin C (MMC) in patients with carcinoma of unknown primary. Eur J Cancer 2003; 39: 1121-1128.

30. Culine S, Lartholary A, Voigt J et al. Cisplatin in combination with either Gemcitabine or Irinotecan in carcinomas of unknown primary site: Results of a randomized Phase II study-trial for the French Study Group on Carcinomas of 
Unknown Primary (GEFCAPI01). J Clin Oncol 2003; 21(18): 3479-3482.

31. Gutzeit A, Antoch G, Kuhl H, Egelhof T et al. Unknown primary tumors: detection with dual-modality PET/CTinitial experience. Radiology 2005; 234 (1): 227-234.

32. Aassar OS, Fischbein NJ, Caputo GR, Kaplan MJ et al. Metastatic head and neck cancer: role and usefulness of FDG PET in locating occult primary tumors. Radiology 1999; 210 (1): 177-181.

33. Lonneux M, Reffad A. Metastases from unknown primary tumor. PET-FDG as initial diagnostic procedure?. Clin Positron Imaging 2000; 3(4): 137-141.

34. Mantaka P, Baum RP, Hertel A, Adams S et al. PET with 2-(F-18)-Fluoro-Deoxy-D-Glucose (FDG) in patients with cancer of unknown primary (CUP): Influence on pa- tients ${ }^{\prime}$ Diagnostic and Therapuetic Management. Cancer Biother Radiopharm 2003; 18(1): 47-58.

35. Wong WL, Saunders M. The impact of FDG PET on the management of occult primary head and neck tumours. Clin Oncol (R Coll Radiol) 2003; 15(8): 461-466.

36. Joshi U, Van der Hoeven JJ, Comans EF, Herder GJ, Teule GJ, HOekstra OS. In search of an unknown primary tumour presenting with extracervical metastases: the diagnostic performance of FDG-PET. Br J Radiol 2004; 77(924): 1000-1006.

37. Nanni C, Rubello D, Castellucci P, Farsad M, Franchi R, Toso S, Barile C, Rampin P, Nibale O, Fanti S. Role of 18F-FDG PET-CT imaging for detection of an unknown primary tumour: preliminary results in 21 patients. Eur $\mathbf{J}$ Nucl Med Mol Imaging 2005; 32(5): 589-592. 